\title{
Elastic wave propagation in nano-composites with random distribution of spherical inclusions
}

\section{Abstract}

In the present study, efforts have been made to theoretically study the diffraction of plane harmonic compressional waves by a spherical nano-inclusion based on the Gurtin-Murdoch surface/interface elasticity theory in which the interface between the nano-inclusion and the matrix is considered as the material surface which has their own mechanical properties. Furthermore, a nano-composite has been considered in order to assess the size effect on the wave propagation characteristics of a plane compressional elastic wave containing the randomly distributed spherical nano-inclusions. Also, the phase velocities and attenuations of $\mathrm{P}$ and $\mathrm{SV}$ elastic waves along with the related dynamic effective elastic properties have been investigated for a wide variety of frequencies and volume fractions.

\section{Keywords}

Nano-composites, Surface/Interface effect, Wave propagation, Size effect.

\author{
Mohammad Rahimzadeh ${ }^{*}$ \\ Ph.D. student, School of Mechanical Engineer- \\ ing, Iran University of Science and Technology, \\ Tehran, Iran, 1684613114
}

Received 14 Nov 2012

In revised form 1 Dec 2012

Author email: mh.rahimzadeh@gmail.com

\section{INTRODUCTION}

Using new materials in high-tech industries entails a vast research in this area. Lately, researchers have shown great interest in the composite materials containing micro- and nano- inclusions. Propagation of elastic waves is a rarely studied subject in the analysis of new materials field. Study of elastic wave propagation in composite materials with nano and micro inclusions can result in a better estimation of dynamic effective elastic properties. The study also facilitates the nondestructive evaluation of the composite materials. It is worth noting that the present study deals with two main subjects. The first subject considers the analysis of elastic wave propagation in elastic environment containing the random distribution of inclusions. The second one focuses on the study of surface effects when the inclusion characteristic size is about nanometers or microns.

Lots of studies have been done regarding the propagation of elastic waves in particulate composites with randomly distributed spherical inclusions via multiple scattering theories. The first im- 
portant theoretical work belongs to Foldy (1945) who studied the multiple scattering of waves by considering the point scatterers and employing a configurationally averaging procedure. Lax (1951) extended Foldy theory considering the anisotropic scatterers. Forward far field scattering amplitude in a single particle wave scattering problem and particle volume fractions have been used in order to complex wave number estimation in these two works. Waterman \& Truell (1961) studied the multiple scattering effects due to a random array of obstacles. They used the backward scattering amplitude as well as the forward scattering amplitude of the single scattering problem in wave dispersion and attenuation relations. The model is valid for sufficiently low densities of obstacles.

To determine the macroscopic elastic module of two-phase composites, a method was proposed by Hill (1965). The method was used as the base for self-consistent approaches in multiple scattering studies of elastic waves. Moon \& Mow (1970) studied the wave propagation in an elastic matrix material containing rigid spherical inclusions via a one-dimensional model. It is assumed that the number of inclusions per unit volume of matrix material is small and is distributed randomly. The coupled equations of motion for both matrix and inclusions have been derived from a variable technique in the work.

Kinra et al. (1980) experimentally investigated the propagation of ultrasonic elastic waves in composite material consisting of spherical glass inclusions distributed randomly in an epoxy matrix. Sayers \& Smith (1983) made a comparison between the single scattering theory of Ying \& Truell (1956) and the experimental results achieved by Kinra (1982). It is shown that the results are in a good agreement in low concentration of inclusions. Using an averaging theory requiring the equivalence of the strain energy and the kinetic energy between the effective medium and the original matrix with inclusions, $\mathrm{Fu} \&$ Sheu (1984) derived the effective module and mass density from a two-phase media. Also, using T-matrix of single scatterer and the quasi-crystalline approximation (QCA), a self-consistent multiple-scattering formulation is presented by Varadan et al. (1985).

Datta et al. (1988) examined the plane longitudinal and shear wave propagation through a composite material containing randomly distributed spherical inclusions, based on Foldy theory. A thin layer with different elastic properties is considered as the interface of matrix and inclusion in this study. Kim et al. (1995) presented an estimation of effective dynamic properties and effective density via a self-consistent method which is similar to the coherent potential approximation in alloy physics. These theoretical results have a better coincidence with the experimental observations of Kinra compared to Ying \& Truell results.

Sato \& Shindo (2003) investigated the multiple scattering of P \& SV harmonic elastic waves in a metal matrix composite with randomly distributed particles with graded interfacial layers using Lax's quasi-crystalline approximation. To study the multiple scattering of plane compressional and shear waves in a medium consisting of spherical inclusions with random distribution, a modified dynamic self-consistent model is proposed by Yang (2003).

The effects of viscoelastic interphase on effective dynamic properties of such composites are studied by Wei \& Haung (2004). The numerical results revealed that the viscosity of interface has a sensible effect on the effective elastic module and effective wave number. The results also indicated that in the low frequency range, the dissipation effect of the interphase is the biggest whereas the effect of multiple scattering dominates in the high frequency range. Based on the generalized selfconsistent model, another approach has been proposed by Kim in order to analyze the elastic wave 
propagation in composites (2004). This approach is similar to GSCM static model in the presence of the elastic wave energy term.

By using both the effective medium and effective field methods, Kanaun et al. (2004) extracted the equations of elastic wave scattering in composites containing of spherical inclusions. They showed that the effective field method has some advantages than the effective medium. Layman et al. (2006) experimentally measured the phase velocity and attenuation of longitudinal and shear wave for random particulate composite. Comparing the experimental observations with two theoretical models including Waterman-Truell and DGSCM showed that both models present a good estimation in low concentration of inclusions while at the higher concentration, the DGSCM is more accurate.

To study the compressional plane wave propagation in composite material having high volume concentration, a new approach was proposed by $\mathrm{Hu}$ et al. (2008). The method used the theory of quasi-crystalline approximation and Waterman's T matrix formulation to deal with multiple scattering problems.

Since the beginning of the present century, there has been great interest to study in the field of nano-materials. As we know the elastic properties of the layers near the surface are different from the bulk layers. From the macroscopic point of view, since the surface to volume ratio is trivial, the difference is negligible, and effective properties are governed by classical bulk relations.

Recently, due to advances made in nanotechnology, nano-devices and nano-materials have become producible. Therefore, the consideration of surface effects will become prominent. One of the most important achievements in the field of nanotechnology is nano-composite production. In these materials, nano-particles are distributed in the matrix that improves the mechanical properties. Due to the considerable ratio of surface to volume in nano-materials, the surface/interface effects will dramatically alter the final effective mechanical properties.

There are considerable works on elastic field determination at a medium with nanoinhomogeneities.

In addition, the above mentioned review shows that many researchers have studied the elastic wave propagation in particulate composites from the macroscopic view. However, contrary to these works, it appeared that rarely works are done in the field of elastic wave propagation in particulate composite with nano- inhomogeneities.

Wang et al. (2006) studied the effects of surface/interface elasticity on the diffraction of plane longitudinal waves by a nano-scale circular hole. Also, he replicated this investigation with a nanoscale spherical cavity and inclusion (2007). He showed that surface/interface effects plays a considerable role in the elastic wave diffraction. In particular, the dynamic stress concentration factor around the nano-inhomogeneity has been examined in the low and high frequency of incident wave.

Considering a cylindrical nano-inhomogeneity, $\mathrm{Ru}$ et al. (2009) investigated the surface/interface effects on the diffraction of plane compressional and shear waves. Hasheminejad and Avazmohammadi (2009) calculated the phase velocity and attenuation of plane compressional and shear elastic waves in unidirectional two-phase medium with transversely isotropic counterparts. They assumed that the nano-sized cylindrical inclusions are distributed randomly in the matrix.

The main objective of this paper was to investigate the elastic wave propagation in a particulate composite containing spherical nano-inclusion with a random distribution. It was assumed that the 
counterparts of two-phase medium were isotropic, and the effective phase velocity and attenuation of elastic wave as well as effective dynamic parameters were analyzed. Recognizing this phenomenon could be helpful in employing new materials for making the industrial devices.

\section{FORMULATIONS}

\subsection{Basic equations in inclusion/matrix interface model}

Equilibrium and constitutive equations inside the matrix and each nano-inclusion are the same as traditional elasticity theory. Assuming no body forces, the equations can be presented as follows:

$$
\begin{gathered}
\sigma_{i j, j}=\rho \frac{\partial^{2} u_{i}}{\partial t^{2}} \\
\sigma_{i j}=2 \mu\left(\varepsilon_{i j}+\frac{\nu}{1-2 \nu} \varepsilon_{k k} \delta_{i j}\right)
\end{gathered}
$$

In which $\sigma_{i j}, \varepsilon_{i j}$ are Cauchy's stress and strain tensors. $\mu$ stands for shear modulus and $\nu$ is Poisson's ratio, $\rho$ and $t$ are mass density and time respectively. Also, the relation between strain tensor and displacement vector is:

$$
\varepsilon_{i j}=\frac{1}{2}\left(u_{i, j}+u_{j, i}\right)
$$

Lately, the Gurtin \& Murdoch model has captured the attention of the researchers due to the consideration of continuum mechanic assumptions. An interface, in surface elasticity theory, is assumed as a thin membrane with trivial mass which is adhered to the abutting bulks with no slipping. Elastic constants based on this theory are different from the bulk ones.

Noting the equilibrium of subsurface, Gurtin \& Murdoch extracted the equilibrium equation of a surface that is called the generalized Yang-Laplace equation. Equilibrium equation at the interface of inclusion and matrix can be described as:

$$
\left[\boldsymbol{\sigma}^{(m)}-\boldsymbol{\sigma}^{(i)}\right] \cdot \mathbf{n}+\nabla_{\mathbf{s}} \cdot \boldsymbol{\sigma}^{s}=0
$$

Where $\mathbf{n}$ denotes the interface normal vector, $-{ }_{s}$ states gradient vector of interface, and subscripts $i, m$ are referred to inclusion and matrix respectively. This equation can be rewritten in the spherical coordinates as following:

Latin American Journal of Solids and Structures 10(2013) $813-831$ 


$$
\begin{gathered}
\sigma_{r r}^{(m)}-\sigma_{r r}^{(i)}=\frac{\sigma_{\theta \theta}^{s}+\sigma_{\varphi \varphi}^{s}}{r} \\
\sigma_{r \theta}^{(m)}-\sigma_{r \theta}^{(i)}=-\left(\frac{1}{r} \frac{\partial \sigma_{\theta \theta}^{s}}{\partial \theta}+\frac{1}{r \sin \theta} \frac{\partial \sigma_{\varphi \theta}^{s}}{\partial \varphi}+\frac{\sigma_{\theta \theta}^{s}-\sigma_{\varphi \varphi}^{s}}{r} \cot \theta\right) \\
\sigma_{r \varphi}^{(m)}-\sigma_{r \varphi}^{(i)}=-\left(\frac{1}{r} \frac{\partial \sigma_{\varphi \theta}^{s}}{\partial \theta}+\frac{1}{r \sin \theta} \frac{\partial \sigma_{\theta \theta}^{s}}{\partial \varphi}+\frac{2 \sigma_{\theta \varphi}^{s}}{r} \cot \theta\right)
\end{gathered}
$$

Constitutive equations in surface/interface elasticity based on Gurtin \& Murdoch model is represented by using the interface free energy and residual stress as bellow:

$$
\boldsymbol{\sigma}^{s}=\frac{\partial \Gamma}{\partial \varepsilon^{s}}+\tau_{0} \mathbf{I}_{\Sigma}=\left[\left(t r \varepsilon^{s}\right)\left(\lambda^{s}+\tau_{0}\right)+\tau_{0}\right] \mathbf{I}_{\Sigma}+2\left(\mu^{s}-\tau_{0}\right) \varepsilon^{s}
$$

Where $\mathbf{I}_{\Sigma}$ Identity the unity tensor of surface/interface, $\mu^{s}, \lambda^{s}$ are surface/interface elastic constants and $\operatorname{tr}\left(\varepsilon^{s}\right)$ is the trace of interface strain tensor. Constitutive equations in spherical coordinate, neglecting of residual surface stress, can be described as:

$$
\begin{gathered}
\sigma_{\theta \theta}^{s}=2 \mu^{s} \varepsilon_{\theta \theta}^{s}+\lambda^{s}\left(\varepsilon_{\theta \theta}^{s}+\varepsilon_{\phi \phi}^{s}\right) \\
\sigma_{\phi \phi}^{s}=2 \mu^{s} \varepsilon_{\phi \phi}^{s}+\lambda^{s}\left(\varepsilon_{\theta \theta}^{s}+\varepsilon_{\phi \phi}^{s}\right)
\end{gathered}
$$

\subsection{Diffraction of longitudinal elastic wave by spherical nano-sized inclusion}

Figure 1 illustrates the geometry of the problem. As the figure suggests, an elastic compressional plane wave that propagates in $z$ direction in an isotropic infinitive medium is diffracted by a spherical nano-inhomogeneity. The infinitive medium and spherical inclusion properties are characterized by subscripts 1 \& 2 respectively. Also, the potential and displacement functions of incident wave have been depicted by superscript $i$, reflected wave by superscript $r$ and refracted by superscript $f$. 


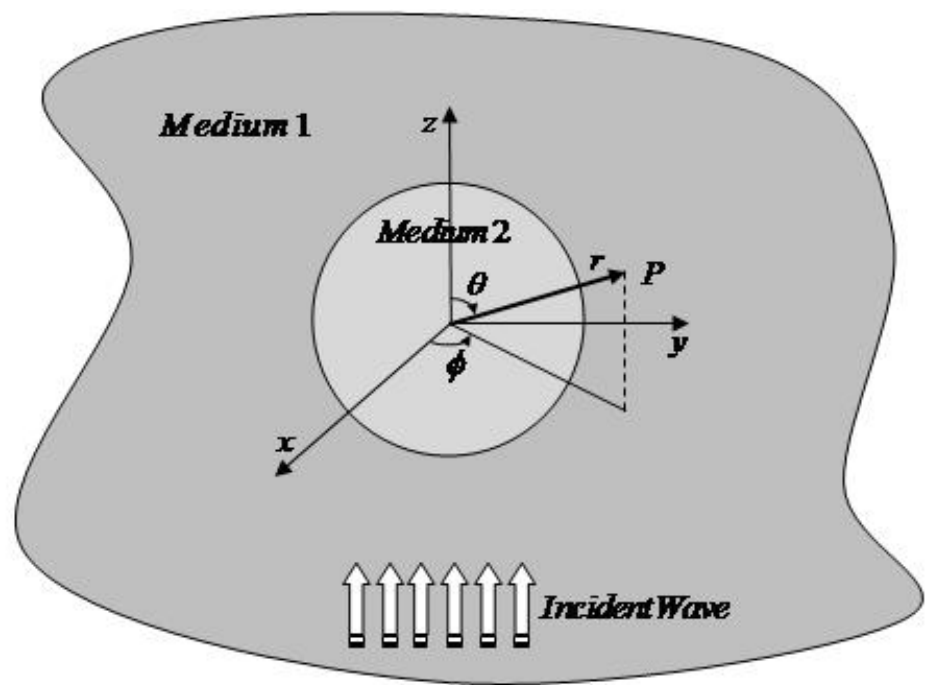

Figure 1 A schematic illustration of $\mathrm{P}$ wave diffraction by a spherical nano-inhomogeneity

When the longitudinal incident wave impinges on the surface of an elastic nano-inclusion, both longitudinal and shear elastic waves are reflected back into the infinitive medium and two waves are refracted into the nano-inclusion.

The incident and two reflected and refracted elastic waves can be represented by:

$$
\begin{gathered}
\varphi^{(i)}=a^{2} \varphi_{0} \sum_{n=0}^{\infty}(2 n+1) I^{n} j_{n}\left(\alpha_{1} r^{\prime}\right) P_{n}(\cos \theta) e^{-I \omega t} \\
\varphi^{(r)}=a^{2} \varphi_{0} \sum_{n=0}^{\infty}(2 n+1) I^{n} A_{n} h_{n}^{(1)}\left(\alpha_{1} r^{\prime}\right) P_{n}(\cos \theta) e^{-I \omega t} \\
\chi^{(r)}=a^{2} \varphi_{0} \sum_{n=0}^{\infty}(2 n+1) I^{n} B_{n} h_{n}^{(1)}\left(\beta_{1} r^{\prime}\right) P_{n}(\cos \theta) e^{-I \omega t} \\
\varphi^{(f)}=a^{2} \varphi_{0} \sum_{n=0}^{\infty}(2 n+1) I^{n} C_{n} j_{n}\left(\alpha_{2} r^{\prime}\right) P_{n}(\cos \theta) e^{-I \omega t} \\
\chi^{(f)}=a^{2} \varphi_{0} \sum_{n=0}^{\infty}(2 n+1) I^{n} D_{n} j_{n}\left(\beta_{2} r^{\prime}\right) P_{n}(\cos \theta) e^{-I \omega t}
\end{gathered}
$$


Where $\alpha_{1}, \alpha_{2}, \beta_{1} \& \beta_{2}$ are compressional and shear wave numbers in the mediums $1 \& 2$ that are normalized by the radius of inclusion. $A_{n}, B_{n}, C_{n} \& D_{n}$ are unknown expansion coefficients that they can be determined by boundary conditions at the nano-inclusion and the medium interface. $\varphi_{0}$ is the amplitude of incident wave and $r^{\prime}=r / a$ is the normalized coordinate. According to the above potential functions, the displacements functions in two medium can be explained as below:

$$
\begin{gathered}
u_{r}^{(1)}=\frac{\varphi_{0} a}{r^{\prime}} \sum_{n=0}^{\infty}(2 n+1) I^{n}\left(Q_{71}^{(1)}+A_{n} Q_{71}^{(3)}+B_{n} Q_{72}^{(3)}\right) P_{n}(\cos \theta) e^{-I \omega t} \\
u_{\theta}^{(1)}=\frac{\varphi_{0} a}{r^{\prime}} \sum_{n=0}^{\infty}(2 n+1) I^{n}\left(Q_{81}^{(1)}+A_{n} Q_{81}^{(3)}+B_{n} Q_{82}^{(3)}\right) \frac{d P_{n}(\cos \theta)}{d \theta} e^{-I \omega t} \\
u_{r}^{(2)}=\frac{\varphi_{0} a}{r^{\prime}} \sum_{n=0}^{\infty}(2 n+1) I^{n}\left(C_{n} W_{71}^{(1)}+D_{n} W_{72}^{(1)}\right) P_{n}(\cos \theta) e^{-I \omega t} \\
u_{\theta}^{(2)}=\frac{\varphi_{0} a}{r^{\prime}} \sum_{n=0}^{\infty}(2 n+1) I^{n}\left(C_{n} W_{81}^{(1)}+D_{n} W_{82}^{(1)}\right) \frac{d P_{n}(\cos \theta)}{d \theta} e^{-I \omega t}
\end{gathered}
$$

The coefficients $Q_{71}^{(1)}, Q_{71}^{(3)}, Q_{72}^{(3)}, Q_{81}^{(1)}, Q_{81}^{(3)} Q_{82}^{(3)}$ and $W_{71}^{(1)}, W_{72}^{(1)}, W_{81}^{(1)}, W_{82}^{(1)}$ are defined in the Appendix.

To formulate the boundary conditions at the interface, the surface/interface stresses shall be calculated first. The components of surface/interface strains on the spherical interface can be described according to the stress components field in the matrix.

$$
\begin{gathered}
\varepsilon_{\theta \theta}^{s}=\frac{1}{E_{1}}\left(\sigma_{\theta \theta}^{(1)}-\nu_{1} \sigma_{r r}^{(1)}-\nu_{1} \sigma_{\phi \phi}^{(1)}\right) \\
\varepsilon_{\phi \phi}^{s}=\frac{1}{E_{1}}\left(\sigma_{\phi \phi}^{(1)}-\nu_{1} \sigma_{r r}^{(1)}-\nu_{1} \sigma_{\theta \theta}^{(1)}\right), \varepsilon_{\theta \phi}^{s}=0
\end{gathered}
$$

Then, surface stress tensor can be calculated by using the constitutive equation in the surface elasticity. Boundary conditions that need to be satisfied on the outer radius of the nano-inclusion include the continuity of displacement components and Yang-Laplace Equilibrium equation. 


$$
\begin{gathered}
u_{r}^{(1)}=u_{r}^{(2)} \\
u_{\theta}^{(1)}=u_{\theta}^{(2)} \\
\sigma_{r r}^{(1)}-\sigma_{r r}^{(2)}=\frac{\sigma_{\theta \theta}^{s}+\sigma_{\phi \phi}^{s}}{a} \\
\sigma_{r \theta}^{(1)}-\sigma_{r \theta}^{(2)}+\frac{\partial \sigma_{\theta \theta}^{s}}{a \partial \theta}+\frac{\cot \theta}{a}\left(\sigma_{\theta \theta}^{s}-\sigma_{\phi \phi}^{s}\right)=0
\end{gathered}
$$

Substituting equations (7) and (10) by the above stress relations, we have:

$$
\begin{gathered}
\sigma_{r r}^{(1)}-\sigma_{r r}^{(2)}=\left(k_{1 s}+k_{2 s}\right)\left[\left(1-\nu_{1}\right)\left(\sigma_{\theta \theta}^{(1)}+\sigma_{\phi \phi}^{(1)}\right)-2 \nu_{1} \sigma_{r r}^{(1)}\right] \\
\sigma_{r \theta}^{(1)}-\sigma_{r \theta}^{(2)}+\left(k_{1 s}-k_{2 s}\right)\left(1+\nu_{1}\right) \cot \theta\left(\sigma_{\theta \theta}^{(1)}-\sigma_{\phi \phi}^{(1)}\right)+\left(k_{1 s}-\nu_{1} k_{2 s}\right) \frac{\partial \sigma_{\theta \theta}^{(1)}}{\partial \theta} \\
+\left(k_{2 s}-\nu_{1} k_{1 s}\right) \frac{\partial \sigma_{\phi \phi}^{(1)}}{\partial \theta}-\left(k_{1 s}+k_{2 s}\right) \nu_{1} \frac{\partial \sigma_{r r}^{(1)}}{\partial \theta}=0
\end{gathered}
$$

In the abovementioned relations, parameters $k_{1 s}$ and $k_{2 s}$ stating the role of surface/interface effects and can be described as following:

$$
\begin{gathered}
k_{1 s}=\frac{2 \mu^{s}+\lambda^{s}}{E_{1} a} \\
k_{2 s}=\frac{\lambda^{s}}{E_{1} a}
\end{gathered}
$$

Unknown coefficients $A_{n}, B_{n}, C_{n} \& D_{n}$ can be specified by subjecting boundary conditions.

\subsection{Multiple scattering approaches}

There are many significant applications for studying about elastic wave propagation through the heterogeneous mediums such as nondestructive evaluation of the composite materials and dynamic effective properties determination. Infact after dynamic properties specification, it's possible to assess the response of inhomogeneous mediums such as particulate composites to various loading conditions like the transient ones as well as fracture and frailer behavior. Elastic wave propagating in 
these materials undergo multiple scattering which results transferring a portion of incident energy to scattered field. So although the two constitutive phases can be nonattenuative, the amplitude of the traveling wave declines and the decay rate is varying with the incident wave frequency. This means that phase velocity and attenuation of the coherent wave as well as dynamic effective properties are frequency dependent. Because of the complexity of random distribution of inclusions, When the inclusions are randomly distributed in the medium, the exact solution does not exist and the approximate solution shall be used. Of the approximate approaches, the self-consistent schemes have considerable benefits. The self-consistent methods are based on the simple assumptions in which the problem of diffraction can be changed from many particles into one particle. There are two basic schemes in self-consistent methods: effective medium and effective field methods.

One of the two basic patterns in self-consistent approach is effective medium. The fundamental hypothesis in the group of the effective medium methods explains that the composite material outside the abutting of the inclusion may be varied by the effective properties of the entire composite that is assumed a homogenous medium (Kanaun 2004). The next self-consistent method is the effective field method. It's assumed that each inclusion is apart in the medium. And the contribution of all other particles is taken into account by the effective field (Kanaun 2005). Of the most popular methods in the set of the effective field method, we can point to Foldy and Waterman-Truell methods which are used in dealing with multiple scattering problems. The geometry of the problem is depicted in Figure 2.

\subsection{A Foldy model in multiple scattering}

Based on the compatible wave treatments, Foldy proposed the first work in the field of multiple scattering of scalar waves by a random distribution of isotropic elastic scatterers. Due to the statistical essence of randomness in scatterers distribution, the average of the physical parameters was taken over a statistical ensemble of all the scatterers.

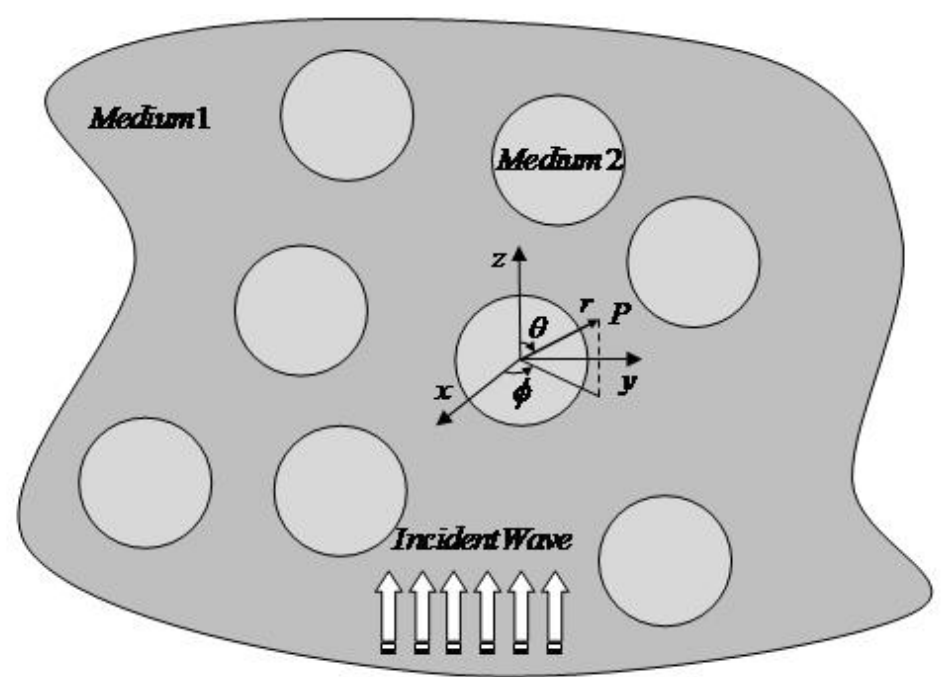

Figure 2 Elastic wave propagation in a composite containing randomly distributed of spherical nano-inhomogeneities 
Foldy realized that the propagation constant of the medium with $n_{0}$ scatterers per unit volume differs from its value $k_{1}$ when no scatterer is in the medium as regards the relation:

$$
\begin{gathered}
\langle k\rangle^{2}=k_{1}^{2}+4 \pi n_{0} f(0) \\
\langle K\rangle^{2}=K_{1}^{2}+4 \pi n_{0} g(0)
\end{gathered}
$$

Where $k_{1}$ and $K_{1}$ denote the wave number of the $\mathrm{P}$ and $\mathrm{S}$ wave in the matrix, and $f(0)$ and $g(0)$ are the far field longitudinal and shear wave scattering amplitude in the forward direction respectively. They are corresponding to the scattering coefficients by:

$$
\begin{gathered}
f(\theta)=\frac{1}{i k_{1}} \sum_{n=0}^{\infty}(2 n+1) A_{n} P_{n}(\cos \theta) \\
g(\theta)=\frac{1}{i 2 K_{1}} \sum_{n=0}^{\infty}(2 n+1)\left(A_{n}+B_{n}\right) P_{n}(\cos \theta)
\end{gathered}
$$

In the case of spherical inclusions in the matrix, the number of scatterers per unit volume $n_{0}$ is related to the concentration $c$ by:

$$
n_{0}=\frac{3 c}{4 \pi a^{3}}
$$

$a$ is the radius of the inclusions. The corresponding wave phase velocity and attenuation can be determined by:

$$
\langle k\rangle=\frac{\omega}{c(\omega)}+i \gamma(\omega)
$$

Where $w$ is the circular frequency, $c(\omega)$ is phase velocity and $\gamma(\omega)$ is attenuation coefficient.

\subsection{B Waterman-Truell model in multiple scattering}

Waterman \& Truell developed the statistical averaging procedure in order to the propagation constant estimation of the inhomogeneous mediums. They proposed a configurationally averaging technique that used the joint probability distribution for the occurrence of a certain configuration in order to calculate the resulted wave over all the configurations. The considered heterogeneous medium contains scatterers whose positions are assumed randomly. In fact, using the physical characteristic of the composed phases, the waterman-Truell model defines an equivalent homogeneous 
medium with the effective complex wave number. The main result of this model is the homogenized wave numbers that have been proposed for the longitudinal waves as:

$$
\left[\frac{\langle k\rangle}{k_{1}}\right]^{2}=\left[1+\frac{2 \pi n_{0} f(0)}{k_{1}^{2}}\right]-\left[\frac{2 \pi n_{0} f(\pi)}{k_{1}^{2}}\right]
$$

And for the shear waves as:

$$
\left[\frac{\langle K\rangle}{K_{1}}\right]^{2}=\left[1+\frac{2 \pi n_{0} g(0)}{K_{1}^{2}}\right]-\left[\frac{2 \pi n_{0} g(\pi)}{K_{1}^{2}}\right]
$$

Where $c$ is the inclusions volume fraction, $f(0)$ and $f(\pi), g(0)$ and $g(\pi)$ are the forward and backward longitudinal and shear scattered wave amplitude of an isolated inclusion respectively.

Waterman \& Truell suggested a criterion to estimate the validity of the proposed relation as below:

$$
\frac{n_{0} \sigma_{\text {scat }}}{k_{1}}<<1
$$

The above conditional relation indicates that when the scattering in the medium increases either by the number of the scatterers or scattering cross section, the result of the model loses its validity.

\section{NUMERICAL RESULTS}

In order to numerically investigate the problem, an infinite medium made of aluminum has been considered. Three states of nano-inhomogeneities are applied to the media that are soft, stiff and cavity. Bulk properties of the composite constituents have been tabulated in the Table.1. Surface properties of aluminum have been taken from Miller and Shenoy (2000) that offer two sets of surface parameters according to the crystallographic structure. Utilized surface parameters have been tabulated in Table.2. The problem is solved for three different states including interface A, interface $\mathrm{B}$ and interface $\mathrm{C}$. In the solution procedure, the size of the spherical nano-inhomogeneities is considered to be equal to $2 \mathrm{~nm}, 5 \mathrm{~nm}$ and $10 \mathrm{~nm}$. 
Table 1 Bulk properties of the composite constituents

\begin{tabular}{|cccc|}
\hline \hline & $r\left(g / \mathrm{cm}^{3}\right)$ & $K(G P a)$ & $m(G P a)$ \\
\hline Matrix & 2.7 & 75.2 & 34.7 \\
Soft Inclusion & 2.7 & 37.6 & 17.3 \\
Stiff Inclusion & 2.7 & 150.4 & 69.4 \\
\hline \hline
\end{tabular}

Table 2 Surface parameters of three considered interfaces

\begin{tabular}{|ccc|}
\hline \hline & $l^{s}(N / m)$ & $m^{s}(N / m)$ \\
\hline Interface A & 3.49 & -6.22 \\
Interface B & 6.84 & -0.376 \\
Interface C & 0 & 0 \\
\hline \hline
\end{tabular}

\subsection{Single scattering results}

Scattering of a compressional elastic wave from the surface of a nano-inhomogeneity within an infinite matrix has been investigated in the first step. Diameter of the spherical nano-inhomogeneity is considered to be equal to $5 \mathrm{~nm}$. The dynamic stress concentration factor is defined as follows:

$$
D S C F_{\theta}=\left|\frac{\sigma_{\theta \theta}^{(1)}}{\sigma_{0}}\right|
$$

Where $\sigma_{\theta \theta}^{(1)}$ is the stress value in infinite elastic material 1 along the surface of nanoinhomogeneity and $\sigma_{0}=\mu_{1} \beta_{1}^{2} \varphi_{0}$ is the stress intensity in the wave propagation direction. The problem is studied for two cases including low and high frequency incident waves.

The results of investigation for interface A and three aforementioned states of nano- inhomogeneities have been depicted in the Figure 3. Also, the figure illustrates the effect of three kinds of considered interfaces with soft nano-inclusion on $D S C F_{\theta}$ for high and low frequency incident wave. As it can be seen in the case of interface $\mathrm{A}$, the value of $D S C F_{\theta}$ around the soft nano-inclusion is more than its value around the stiff's one. In the case of low frequency incident wave, the maximum and minimum values of $D S C F_{\theta}$ respectively happen around $\theta=\pi / 2$ and $\theta=0, \pi$. There is a different situation in the case of the nano-cavity, as the maximum value of $D S C F_{\theta}$ is shifted toward $\theta=\pi$ and its minimum value can be observed around $\theta=\pi / 2$. As it can be seen for the stiff nano-inclusion the value of $D S C F_{\theta}$ is greater than nano-cavity except in $\theta=\pi$. 

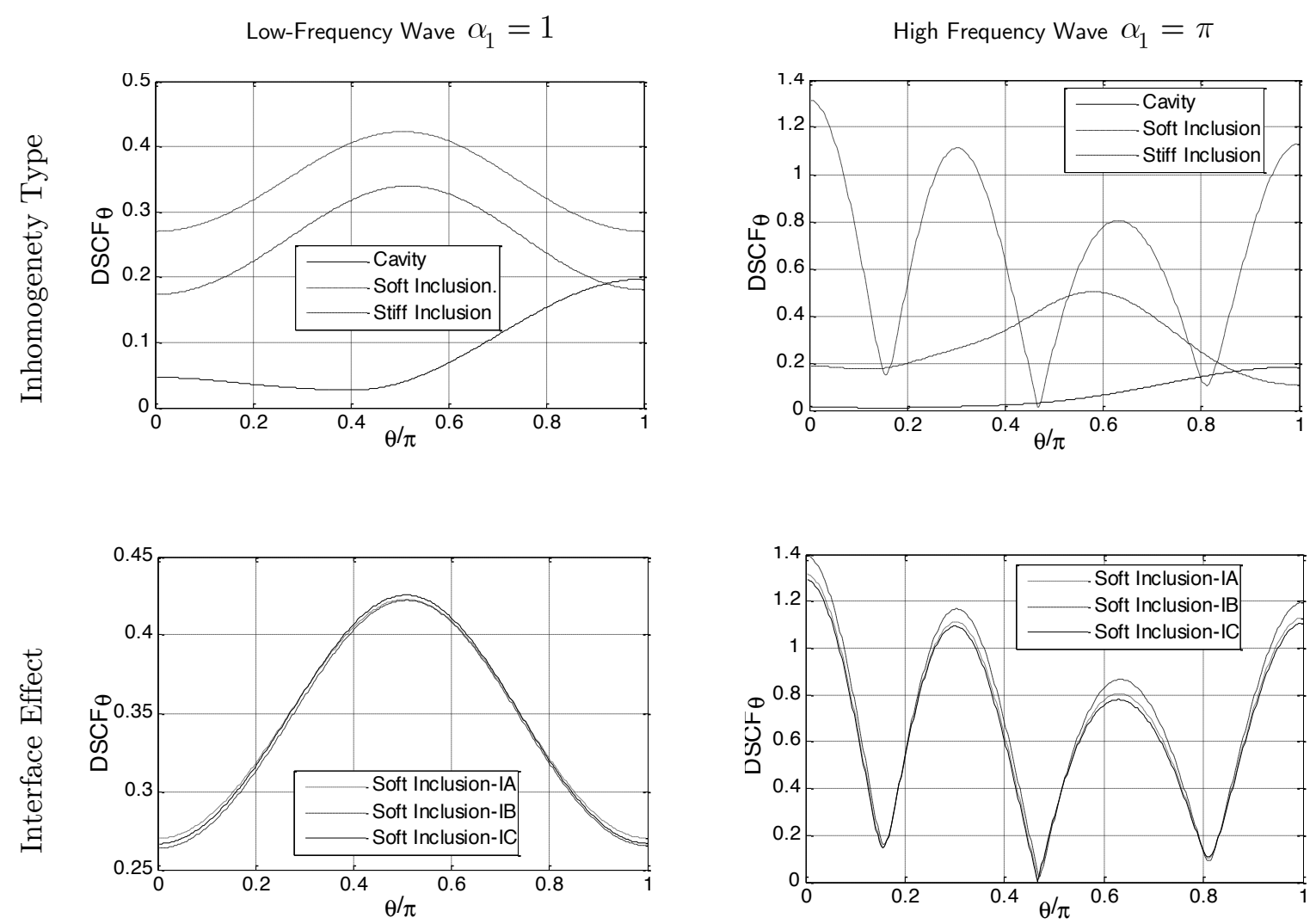

Figure 3 Distribution of $D S C F_{\theta}$ according to inhomogenety type and interface effect in low and high frequency incident wave

For the case of high frequency incident wave, due to the equity in wave length and nanoinhomogeneity diameter, scattering phenomenon is dominant. In this case, $D S C F_{\theta}$ isn't symmetric around the y axis.

In the case of stiff nano-inclusion and nano-cavity, the maximum of this parameter will occur in the incident side. Whereas, in the case of soft nano-inclusion, it can be seen in $\theta=0$. Multiple peaks in the values of circumferential stresses around the nano-inclusion surface can be observed. This phenomenon can be explained to take place as the result of the interference of incident and reflected waves. In the second part of this figure, there is a comparison between the $D S C F_{\theta}$ values for soft nano-inclusion in three different interfaces. The results show that the interface effects can increase or decrease the values of $D S C F_{\theta}$. Such a condition can be seen in the high frequency incident wave. 


\subsection{Multiple Scattering results}

The effect of interface properties on variation of phase velocities and attenuations of $\mathrm{P}$ and SV waves with the normalized frequency has been illustrated in the Figure 4. The results have been obtained according to Watreman-Truell model for a nano-composite at the concentration $\mathrm{c}=15 \%$. By noting the results, it is obvious that the wave's phase velocities and attenuations are remarkably influenced by interface effects. The obtained curves have a similar trend in the considered frequency range. Increasing the size of nano-inclusions, the obtained results will converge to the results for interface $\mathrm{C}$ ignoring the interface effects. The behavior can be explained by increasing the absolute value of $k_{1 s}=\left(\lambda^{s}+2 \mu^{s}\right) / E_{1} a$. For the composite with soft nano-inclusions, the phase velocity and attenuation curves are more influenced by interface effects compared to stiff ones. Infact as the inclusions are considered to have more rigidity, the interface effects can be neglected. The influence of interface effects on the attenuation curves in low frequency incident wave is hardly noticeable and for the composite with stiff nano-inclusions is negligible.

Figures 5 illustrate the interface effects on $\mathrm{P}$ and SV wave phase velocity and attenuation curves in respect to different volume fractions in $\alpha_{1}=\pi$. The main observed result is that in low volume fractions, the interface effects have no considerable influence on the phase velocity and attenuation curves. Increasing the volume fraction, this influence will be remarkable. It should be noted that the influence of interface effects on these curves for composites with stiff nano-inclusion is ignorable.

The effect of size of nano-inclusion on normalized effective dynamic elastic properties for different interfaces has been illustrated in Figure 6. The results in this figure have been obtained when $\mathrm{c}=30 \%$ and $\alpha_{1}=1$. The effective dynamic elastic properties are normalized according to the elastic constants without considering interface effects (Interface C). Increasing the diameter of inclusions, the effective dynamic elastic properties converge to the results obtained for interface C. This statement can be deduced from Figure 6. Also, these effects are more considerable for composites with soft nano-inclusions.

\section{CONCLUSIONS}

Using the surface/interface theory, the present study investigated the diffraction of plane $\mathrm{P}$ wave by a nano-sized spherical inclusion. Also, the influence of material bulk properties and interface properties on the dynamic stress concentration around the inclusion was examined. It has been found that the surface/interface elasticity significantly affects the elastic scattering field when the inclusion size shrinks to nanometers. Furthermore, we have investigated the effective wave field properties propagating in an isotropic medium with randomly distributed spherical nano-inhomogeneities based on the Waterman-Truell model. It has been observed that the interface properties have considerable effects on the phase velocities when the inhomogeneities are in nanometers scale. Additionally, the effect decreases when the size of inclusions increases. The interface stress effects on the phase velocities are noticeably higher on the nano-composites with soft nano-inclusions. It has been observed that the attenuation curves are negligible sensitive to the size effect phenomenon.

Latin American Journal of Solids and Structures 10(2013) $813-831$ 

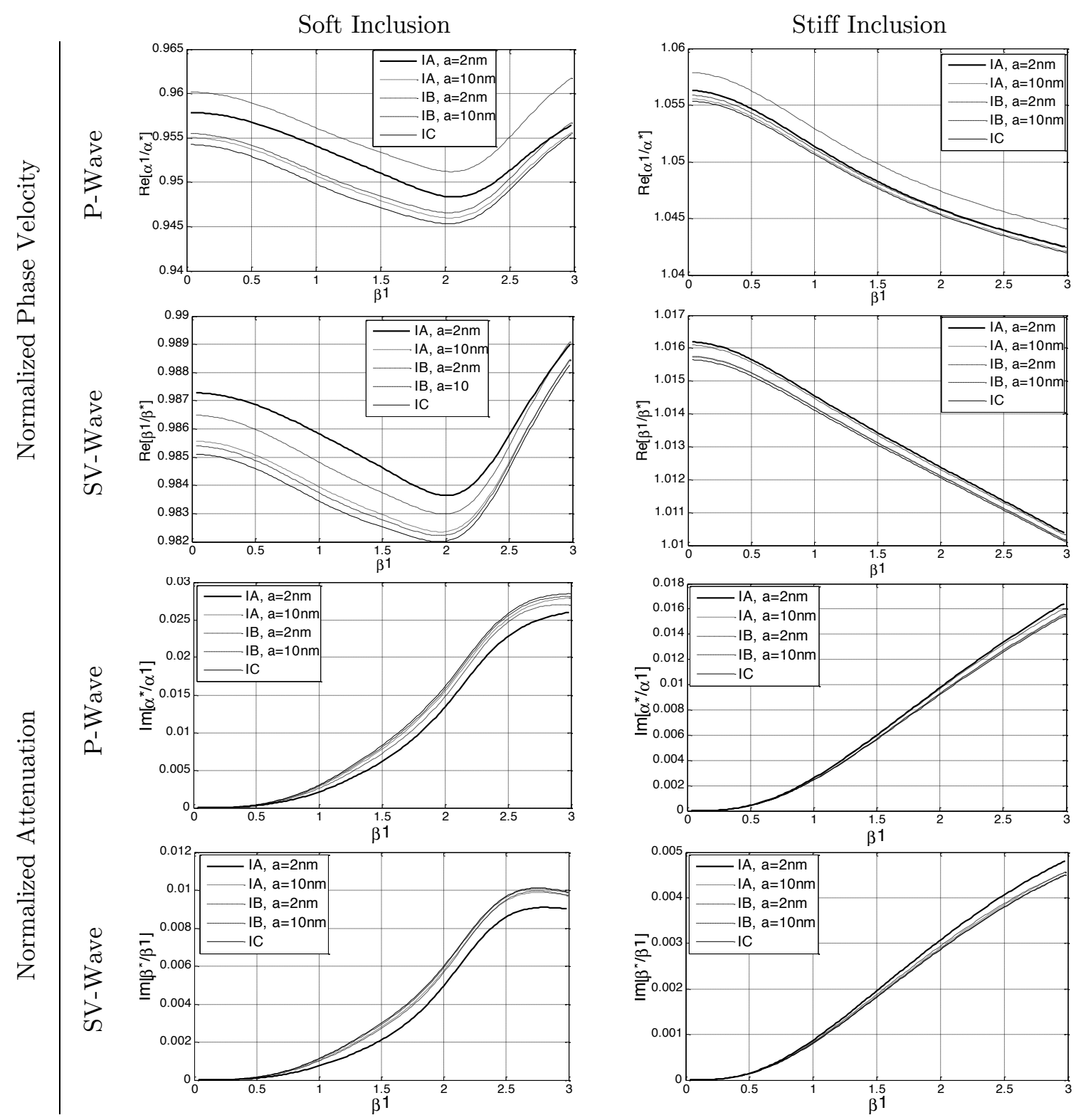

Figure 4 Interface effects on phase velocity and attenuation of $\mathrm{P}$ and SV waves calculated from Waterman-Truell theory for concentration $c=15 \%$ and the selected interface types 
Soft Inclusion
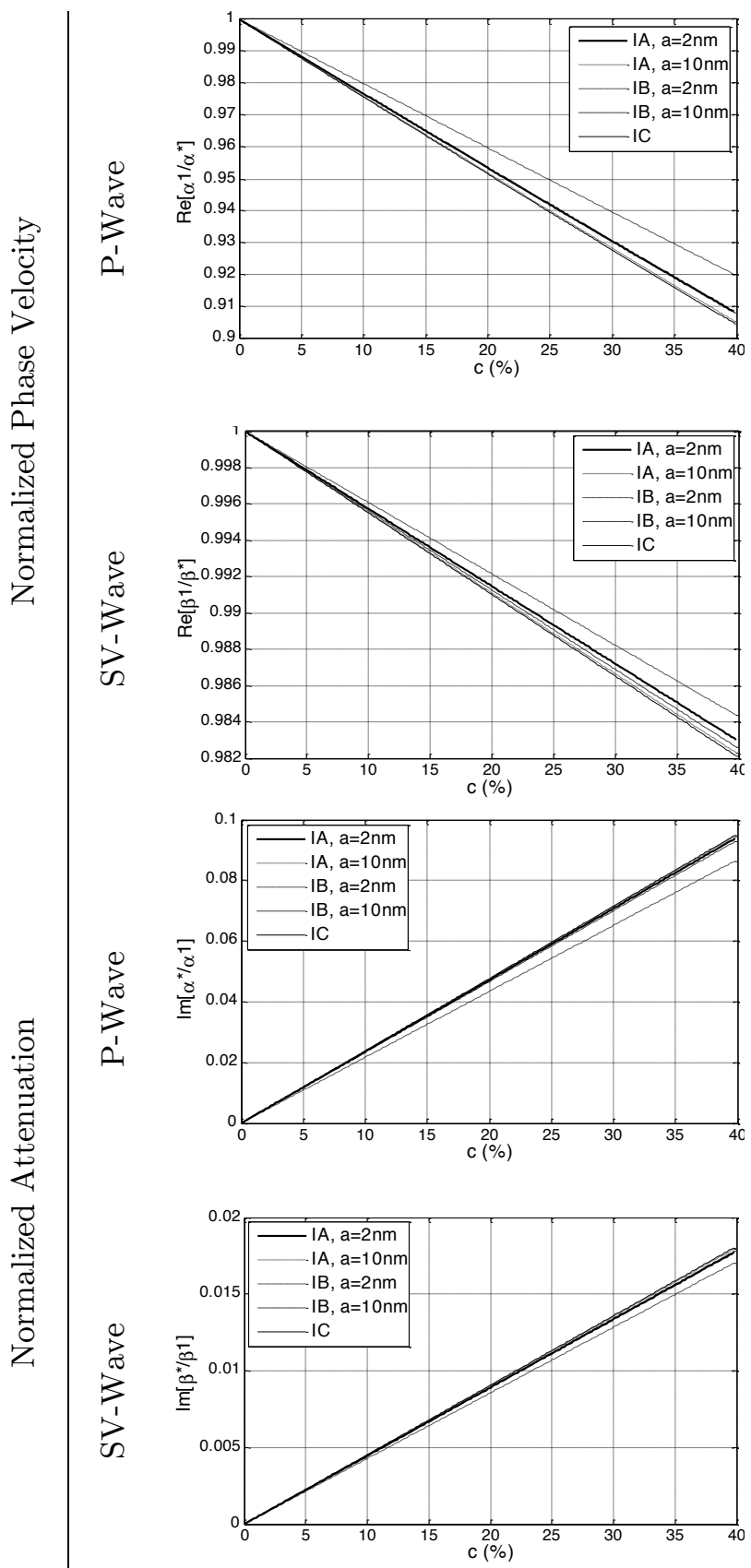

Stiff Inclusion
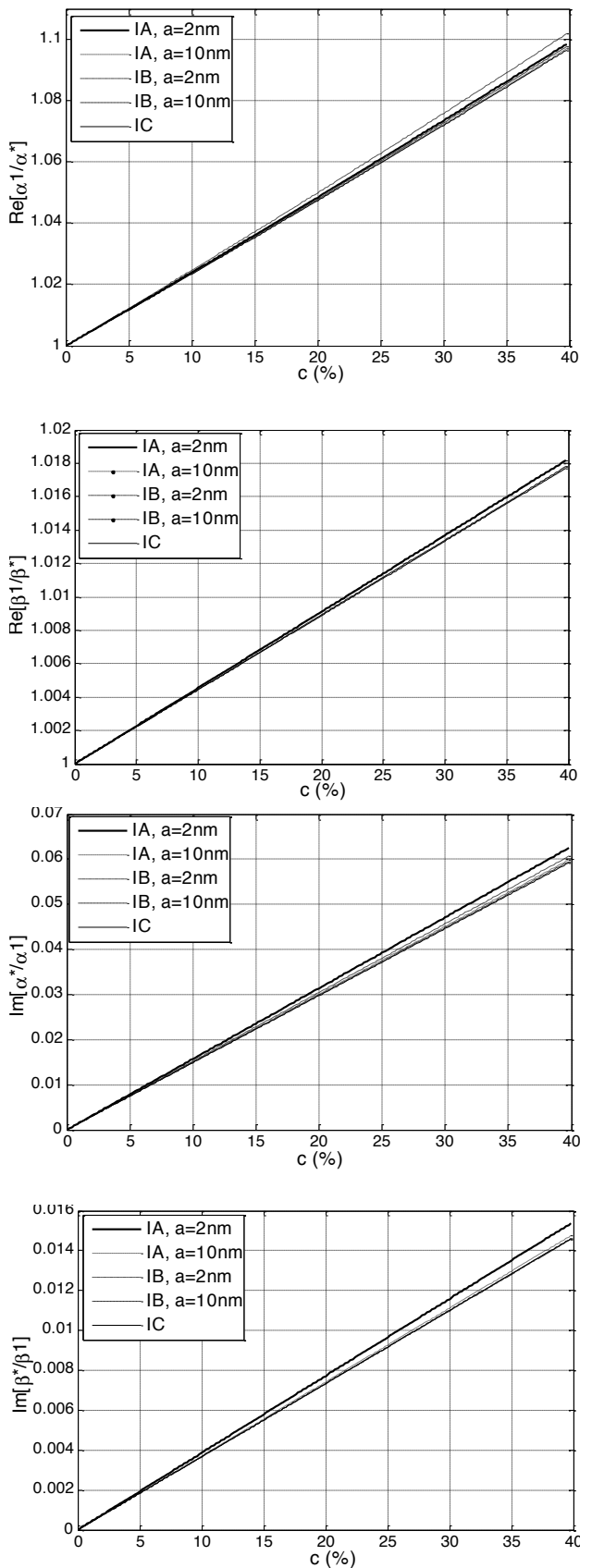

Figure 5 Interface effects on phase velocity and attenuation of P and SV waves calculated from Waterman-Truell theory for $\alpha_{1}=\pi$ and the selected interface types 

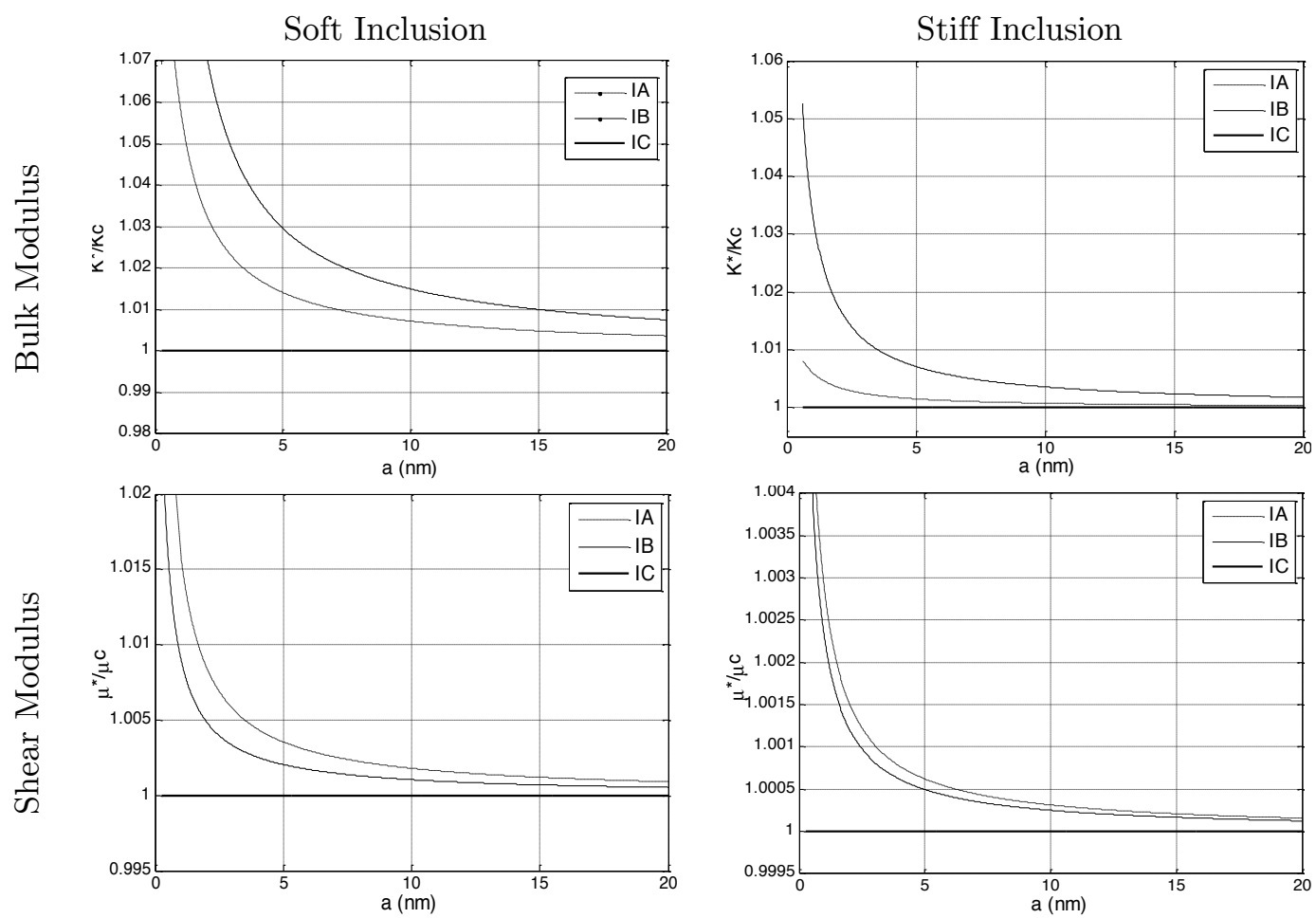

Figure 6 Interface effects on dynamic effective elastic moduli for $\mathrm{c}=30 \%$ and $\alpha_{1}=1$

\section{References}

Foldy L. L., The Multiple Scattering of Waves, Phys. Rev., 67:107-119, 1945.

Lax M., Multiple Scattering of Waves, Rev. Mod. Phys., 23:287-310, 1951.

Waterman P. C., Truell R., Multiple Scattering of Elastic Waves, J. Math. Phys., 2:512-537, 1961.

Hill, R.,A Self Consistent Mechanics of Composite Materials, J. Mech. Phys. Solids, 13:213-222, 1965.

Moon F.C., Mow C.C.,Wavep ropagatiino an c omposmitea terial containindgi sperserdig ids phericainl clusions, Rand Corp. Report, RM-6139-PR, Santa Monica, CA (1970).

Kinra V.K., Petraitis M.S., Datta S.K.,Ultrasonic wave propagation in a random particulate composite, Int. J. Solids Struct., 16:301-312, 1980.

Sayers C.M., Smith R.L, Ultrasonic velocity and attenuation in an epoxy matrix containing lead inclusions, J. Phys. D: Appl. Phys, 16:1189-1194, 1983.

Ying C. F., Truell R., Scattering of a plane longitudinal wave by a spherical obstacle in an isotropically elastic solid, J. Appl. Phys., 27:1086-1097, 1956.

Kinra V.K., Ker E., Datta S.K., Influence of particle resonance on wave propagation in a random particulate composite, Mech. Res. Commun., 9:109-114, 1982.

Fu L.S., Sheu Y.C, Ultrasonic wave propagation in two-phase media: Spherical inclusions, composite Structures, 2:289-303, 1984.

Varadan V.K., Ma Y., Varadan V.V., A multiple scattering theory for elastic wave propagation in discrete random media, J. Acoust. Soc. Am., 77:375-385, 1985.

Datta S.K., Ledbetter H.M., Shindo Y., Shah A.H., Phase velocity and attenuation of plane elastic waves in a particle-reinforced composite medium, Wave Motion, 10:171-182, 1988.

Kim J.Y., Ih J.G., Lee B.H., Dispersion of elastic waves in random particulate composites, J. Acoust. Soc. Am. , 97:1380-1388, 1995. 
Sato H., Shindo Y., Multiple scattering of plane elastic waves in a particle-reinforced-composite medium with graded interfacial layers, Mech. Mater., 35:83-106, 2003.

Yang R.B., A Dynamic Generalized Self-Consistent Model for Wave Propagation in Particulate Composites, J. Appl. Mech., 70:575-582, 2003.

Wei P.J., Huang Z.P., Dynamic effective properties of the particle-reinforced composites with the viscoelastic interphase, Int. J. Solids Struct., 41:6993-7007, 2004.

Kim J.-Y., On the generalized self-consistent model for elastic wave propagation in composite materials, Int. J. Solids Struct., 41:4349-4360, 2004.

Kanaun S.K., Levin V.M., Sabina F.J., Propagation of elastic waves in composites with random set of spherical inclusions (effective medium approach), Wave Motion, 40:69-88, 2004.

Layman C., Murthy N.S., Yang R.B., Wu J., The interaction of ultrasound with particulate composites, J. Acoust. Soc. Am., 119:1449-1456, 2006.

Hu C., Fang X., Huang W., Dynamic effective properties of matrix composite materials with high volume concentrations of particles, Arch. Appl. Mech., 78:177-190, 2008.

Gurtin M.E., Ian Murdoch A., A continuum theory of elastic material surfaces, Arch. Ration. Mech. Anal., 57:291323,1975 .

Wang G.F., Wang T.J., Feng X.Q., Surface effects on the diffraction of plane compressional waves by a nanosized circular hole, Appl. Phys. Lett., 89:231923, 2006.

Wang G.F., Diffraction of plane compressional wave by a nanosized spherical cavity with surface effects, Appl. Phys. Lett., 90:211907, 2007.

Wang G.F., Feng X.Q., Yu S.W., Interface effects on the diffraction of plane compressional waves by a nanosized spherical inclusion, J. Appl. Phys., 102:043533, 2007.

Ru Y.,Wang G.F., Wang T.J., Diffractions of elastic waves and stress concentration near a cylindrical nano-inclusion incorporating surface effect, Journal of Vibration and Acoustics, 131: Art. 061011, 2009.

Hasheminejad S., Avazmohammadi R., Size-dependent effective dynamic properties of unidirectional nanocomposites with interface energy effects, Composites Science and Technology, 69 (15-16):2538-2546, 2009.

Kanaun S.K., Levin V.M., Propagation of shear elastic waves in composites with random set of spherical inclusions (effective field approach), international journal of solids and structures, 42:3971-3997, 2005. 


\section{Appendix}

The coefficients $Q_{71}^{(1)}, Q_{71}^{(3)}, Q_{72}^{(3)}, Q_{81}^{(1)}, Q_{81}^{(3)} Q_{82}^{(3)}$ and $W_{71}^{(1)}, W_{72}^{(1)}, W_{81}^{(1)}, W_{82}^{(1)}$ in equation (9) are:

$$
\begin{gathered}
Q_{71}^{(1)}=n j_{n}\left(\alpha_{1} r^{\prime}\right)-\alpha_{1} r^{\prime} j_{n+1}\left(\alpha_{1} r^{\prime}\right) \\
Q_{71}^{(3)}=n h_{n}^{(1)}\left(\alpha_{1} r^{\prime}\right)-\alpha_{1} r^{\prime} h_{n+1}^{(1)}\left(\alpha_{1} r^{\prime}\right) \\
Q_{72}^{(3)}=n(n+1) h_{n}^{(1)}\left(\beta_{1} r^{\prime}\right) \\
Q_{81}^{(1)}=j_{n}\left(\alpha_{1} r^{\prime}\right) \\
Q_{81}^{(3)}=h_{n}^{(1)}\left(\alpha_{1} r^{\prime}\right) \\
Q_{82}^{(3)}=(n+1) h_{n}^{(1)}\left(\beta_{1} r^{\prime}\right)-\beta_{1} r^{\prime} h_{n+1}^{(1)}\left(\beta_{1} r^{\prime}\right) \\
W_{71}^{(1)}=n j_{n}\left(\alpha_{2} r^{\prime}\right)-\alpha_{2} r^{\prime} j_{n+1}\left(\alpha_{2} r^{\prime}\right) \\
W_{72}^{(1)}=n(n+1) j_{n}\left(\beta_{2} r^{\prime}\right) \\
W_{81}^{(1)}=j_{n}\left(\alpha_{2} r^{\prime}\right) \\
W_{82}^{(1)}=(n+1) j_{n}\left(\beta_{2} r^{\prime}\right)-\beta_{2} r^{\prime} j_{n+1}\left(\beta_{2} r^{\prime}\right)
\end{gathered}
$$

